\title{
The effect of cigarette smoke extract on thrombomodulin- thrombin binding: an atomic force microscopy study
}

\author{
WEI YuJie ${ }^{1 \dagger}$, ZHANG XueJie ${ }^{2 \dagger}$, XU Li $^{2}$, YI ShaoQiong ${ }^{3}$, LI Yi $^{1}$, FANG XiaoHong ${ }^{2 *}$ \\ \& LIU HuiLiang ${ }^{1 *}$ \\ ${ }^{1}$ Department of Cardiology, the General Hospital of Chinese People's Armed Police Forces, Beijing 100039, China; \\ ${ }^{2}$ Beijing National Laboratory for Molecular Sciences, CAS Key Laboratory of Molecular Nanostructure and Nanotechnology, Institute of \\ Chemistry, Chinese Academy of Sciences, Beijing 100190, China; \\ ${ }^{3}$ Beijing Institute of Biotechnology, Academy of Military Medical Sciences, Beijing 100071, China
}

Received June 16, 2012; accepted August 7, 2012

\begin{abstract}
Cigarette smoking is a well-known risk factor for cardiovascular disease. Smoking can cause vascular endothelial dysfunction and consequently trigger haemostatic activation and thrombosis. However, the mechanism of how smoking promotes thrombosis is not fully understood. Thrombosis is associated with the imbalance of the coagulant system due to endothelial dysfunction. As a vital anticoagulation cofactor, thrombomodulin (TM) located on the endothelial cell surface is able to regulate intravascular coagulation by binding to thrombin, and the binding results in thrombosis inhibition. This work focused on the effects of cigarette smoke extract (CSE) on TM-thrombin binding by atomic force microscopy (AFM) based single-molecule force spectroscopy. The results from both in vitro and live-cell experiments indicated that CSE could notably reduce the binding probability of TM and thrombin. This study provided a new approach and new evidence for studying the mechanism of thrombosis triggered by cigarette smoking.
\end{abstract}

cigarette smoke extract (CSE), thrombin, thrombomodulin, AFM, single-molecule force spectroscopy, protein-protein interaction

Citation: Wei Y J, Zhang X J, Xu L, et al. The effect of cigarette smoke extract on thrombomodulin-thrombin binding: an atomic force microscopy study. Sci China Life Sci, 2012, 55: 891-897, doi: 10.1007/s11427-012-4383-y

Epidemiological and medical investigations have demonstrated that exposure to cigarette smoking is an independent risk factor for cardiovascular disease [1,2], as it causes significant endothelial dysfunction, an inflammatory state, and triggers coronary thrombosis [3-5]. Evidence from autopsy and clinical studies also showed that smoking is critical to arterial thrombosis via a direct toxic effect on the injured endothelium and endothelial cells, resulting in alterations of the haemostatic system and an increase in platelet reactivity which is relevant for the pathogenic effect of atherothrom-

$\dagger$ Contributed equally to this work

*Corresponding author (email: xfang@iccas.ac.cn; 1hl518@vip.sina.com) bosis [6-8].

To better understand the molecular mechanism of the increased risk for chronic dysfunction from smoking, cigarette smoking extract (CSE) is widely used to mimic the tobacco dust environment induced by human smoking [9-11]. Edirisinghe et al. [12] found CSE impaired vascular endothelial growth factor (VEGF-) and fluid shear stress (FSS-) mediated endothelial cell function, and had important correlation with cardiovascular diseases associated with endothelial dysfunction. Other studies showed that CSE could induce a dysfunction on fibrinolytic activity in human umbilical vein endothelial cells [13], and consequently a hypercoagulable state for thrombosis. Normal endothelium has 
the ability for self-protection by generating a number of substances, including various antiagglutinating and anticoagulant factors such as prostacyclin and thrombomodulin (TM) [14]. As a crucial anticoagulation cofactor, TM is constitutively expressed on the endothelial cell surface. TM can form a high affinity 1:1 complex with thrombin. The complex of thrombin/TM has several important roles including activation of protein $\mathrm{C}$ and thrombin-activatable fibrinolysis inhibitor (TAFI), anti-inflammatory, and anticoagulation [15-17]. Whether CSE could affect thrombin/TM binding and induce intravascular thrombosis through the thrombin/TM pathway is as yet unclear.

Atomic force microscopy (AFM) has been developed to be a powerful tool in the study of biomolecular interactions via single molecular force spectroscopy (SMFS) $[18,19]$. Encouraging results have been achieved on measuring the binding forces at the molecular level in buffer solution and near physiological conditions for protein/DNA, DNA/DNA, ligand/receptor and other biomolecule pairs [20-24]. These SMFS studies have offered valuable information for the investigation into the molecular basis for biomolecular recognition, cellular adhesion, and signal transduction. This method has also been successfully applied to study the effect of clinically drugs and drug candidates on the important biomolecular interactions [20,22,25].

In this work, we applied AFM SMFS combined with fluorescence microscopy to study the effect of CSE on the interaction of thrombin and TM in vitro and with living cells. The study adds to the understanding of CSE induction of thrombosis, showing that CSE inhibits thrombin/TM binding. For the first time the mechanism of action of CSE on thrombosis has been studied at the single molecular level.

\section{Materials and methods}

\subsection{Materials}

Thrombin from human plasma, TM (human recombination), poly-D-lysine (Mw: 70000-150000), (3-Aminopropyl) triethoxysilane (APTES) and (3-Mercaptopropyl) trimethoxysilane (MPTMS) were all purchased from Sigma-Aldrich (Shanghai, China). The monoclonal antibody of TM was obtained from Abcam (Hong Kong, China). N-hydroxysuccinimide-polyethylene glycol-maleimide (NHS-PEGMAL, Mw: 3400) was purchased from Laysan Bio (Arab, Alabama, USA). Vectors used in plasmid construction for pMD18t and pEGFP-N1 were purchased from TaKaRa (Dalian, China) and Invitrogen (New York, USA) respectively. Lipofectamine was also obtained from Invitrogen. HEPES was obtained from Sino-American Biotech. (Los Angeles, USA). The reagents MEM and Opti-MEM were bought from Gibco, USA, while fetal bovine serum was purchased from Hyclone (Omaha, USA). Milli-Q purified water (18.2 M $\Omega$ ) was used for all experiments. Other reagents used in the experiments were all of analytical grade.

\subsection{Plasmid construction and cell culture}

The complete human TM cDNA fragment was amplified by RT-PCR using specific primers corresponding to the sequences (GenBank No. NM_000361.2; GenBank GI: 40288292). Primers were 5'-CGAAGCTTATGCTTGGGGTCCTGGTC-3' (forward), and 5'-ATGGATCCGAGTCTCTGCGGCGTCGC-3' (reverse).

The Hind III-digested positions were contained in all forward primers, whereas the Bam H I-digested positions were contained in the reverse primers. After RT-PCR and restriction enzyme digestion, products were separated and purified, followed by their insertion into the $\mathrm{pMD} 18 \mathrm{t}$ vector and then digested and inserted into the pEGFP-N1 vector with the same enzyme digestion. Construction of the plasmids was confirmed using sequencing.

COS-7 cells were used in this study, and were cultured in 35-mm tissue culture dishes with MEM, containing 10\% fetal bovine serum in a humidified $5 \% \mathrm{CO}_{2}$ atmosphere at $37^{\circ} \mathrm{C}$. Transient transfection was performed with lipofectamine 2000 and $4 \mu \mathrm{g} \mathrm{mL}^{-1}$ plasmids (TM-GFP or pEGFP-N1) in Opti-MEM when cells were at 70\%-80\% confluence. After $24 \mathrm{~h}$ incubation, the cells were washed twice with serum-free medium and the culture solution was changed to serum-free MEM containing $10 \mathrm{mmol} \mathrm{L}^{-1}$ HEPES for microscopy observation and force measurement.

\subsection{Cigarette smoke extract (CSE) preparation}

CSE was prepared using a previously reported method with slight modifications [26,27]. Two cigarettes (each containing $1.1 \mathrm{mg}$ of nicotine, $13 \mathrm{mg}$ of carbon monoxide and $12 \mathrm{mg}$ of tar) without filters, were smoked consecutively through a facility driven by a $50 \mathrm{~mL}$ injection syringe with a constant airflow. The smoke was bubbled through $50 \mathrm{~mL}$ phosphate buffered solution (PBS, $10 \mathrm{mmol} \mathrm{L}{ }^{-1} \mathrm{Na}_{2} \mathrm{HPO}_{4}$, $1.8 \mathrm{mmol} \mathrm{L}{ }^{-1} \mathrm{KH}_{2} \mathrm{PO}_{4}, 2.7 \mathrm{mmol} \mathrm{L}{ }^{-1} \mathrm{KCl}, 140 \mathrm{mmol} \mathrm{L}^{-1}$ $\mathrm{NaCl}, \mathrm{pH}=7.4$ ) and controlled with a three-way cock, thus mimicking the gas fluid pathway during the smoking process in the human lung. The obtained CSE solution was filtered through a $0.22-\mu \mathrm{m}$ filter to remove bacteria and large impurities. The $\mathrm{pH}$ of the CSE-PBS buffer was adjusted to a final value of 7.4. The concentration of this obtained stock solution was defined as $100 \%$ CSE, and the solution was diluted in PBS buffer to a final concentration required in the experiment. The CSE solution was prepared immediately before each experiment.

\subsection{AFM tip preparation}

AFM silicon nitride $\left(\mathrm{Si}_{3} \mathrm{~N}_{4}\right)$ tips (NP-10, Veeco, Santa Barbara, CA, USA) were used in all the experiments. Chemical modification of AFM tips for force measurement was carried out according to previously reported procedures [20-24]. The cantilevers were placed in a plasma reactor 
(PDC-32G-2, Harrick Scientific Co., NY, USA) to get clean and hydroxyl surfaces. The cleaned tips were incubated in a solution of $1.0 \%(\mathrm{v} / \mathrm{v})$ MPTMS in toluene for $1 \mathrm{~h}, 1.0 \mathrm{mg}$ $\mathrm{mL}^{-1}$ NHS-PEG-MAL in DMSO for $2 \mathrm{~h}$, and protein (e.g., thrombin) solution $\left(2 \mu \mathrm{g} \mathrm{mL}{ }^{-1}\right.$ in PBS) for $0.5 \mathrm{~h}$, sequentially. After every reaction step, tips were washed using corresponding solvents sufficiently to remove all the un-reacted reagents. After final rinsing with PBS, the protein-modified tips were stored in PBS at $4^{\circ} \mathrm{C}$ until use.

\subsection{Silicon wafers preparation}

Single-crystal silicon wafers were cut into $1 \mathrm{~cm} \times 1 \mathrm{~cm}$ squares and modified with the protein TM-ECD as previously reported $[21,23,24]$. The wafers were first cleaned and hydroxized through treatment with chloroform, $\mathrm{HF}$ acid, alkaline solution $\left(\mathrm{NH}_{4} \mathrm{OH} / \mathrm{H}_{2} \mathrm{O}_{2} / \mathrm{H}_{2} \mathrm{O}=1: 1: 5\right.$, v/v) in an ultrasonicator. Piranha solution $\left(98 \% \mathrm{H}_{2} \mathrm{SO}_{4} / \mathrm{H}_{2} \mathrm{O}_{2}=7: 3\right.$, v/v) was heated separately to $90^{\circ} \mathrm{C}$ to generate $\mathrm{Si}-\mathrm{OH}$ groups. The cleaned wafers were transferred to a solution of $1.0 \%$ $(\mathrm{v} / \mathrm{v})$ APTES in toluene, incubated for $2 \mathrm{~h}$ at room temperature to obtain an amino surface, and then thoroughly and consequently rinsed with toluene, acetone and alcohol. Wafers were then dried with nitrogen gas. The silanizing silicon wafers were transferred into TM-ECD solution $(1 \mu \mathrm{g}$ $\mathrm{mL}^{-1}$ ) for $3 \mathrm{~h}$ at room temperature, and then cleaned with PBS buffer, and kept at $4^{\circ} \mathrm{C}$ until use.

\subsection{Single-molecule force spectroscopy}

The measurement of force between the thrombin modified AFM tips and the TM-ECD modified silicon substrate or TM-GFP expressed on the living cells was carried out using PicoSPM with a PicoScan 3000 controller and a larger AFM scanner. The scanner was mounted on a fluorescence microscope (Olympus $\times 71$, Japan). The fluorescence microscope was equipped with a mercury air lamp (OSRAM, Germany) and $10 \times / 40 \times$ objectives. A U-MWIB2 filter set (Ex: BP 460-490 nm, Em: LP 510 nm, Olympus, Japan) was used for GFP imaging. The single molecule force spectrum was measured at the loading rate of $10^{3}-10^{4} \mathrm{pN} \mathrm{s}^{-1}$ with a soft cantilever $\left(0.06 \mathrm{~N} \mathrm{~m}^{-1}\right)$. The calibrated spring constants of the protein modified tips were measured using the thermal noise method, ranging from 0.069 to $0.078 \mathrm{~N} \mathrm{~m}^{-1}$.

\section{Results and discussion}

\subsection{Measurement of single-molecule binding force of thrombin-TM on silicon wafers}

We first measured the binding force of thrombin and TM in vitro using AFM single molecule force spectroscopy. With chemical modification, we coupled thrombin onto the AFM tips via a PEG linker (averaged length approximately $30 \mathrm{~nm}$ ) and the extracellular domain of TM (TM-ECD) onto the silicon substrates. The PEG linker was used to keep the protein in a more flexible state and differentiate specific binding from nonspecific interaction [22,24]. The protein densities of both thrombin modified on tips and TM-ECD on silicon substrate were controlled to be low, so that only one pair of thrombin/TM-ECD molecules could be formed during the force measurement. The rupture forces of the complex of thrombin/TM-ECD were detected when the tip and silicon substrate were brought into and out of contact. Forces were measured with the same loading rates $10^{3}-10^{4}$ $\mathrm{pN} \mathrm{s}^{-1}$. The representative force curves measured for thrombin/TM-ECD are shown in Figure 1A. The peak appearing around 20-50 $\mathrm{nm}$ away from separation of the tip and substrate (Figure 1A(a)) was considered as the specific rupture of thrombin/TM-ECD, while peaks randomly seen after the separation point of the AFM tip and substrate were caused by nonspecific interaction (Figure 1A(b)). Figure $1 \mathrm{~A}(\mathrm{c})$ indicates that, when TM-ECD on the substrate was covered by binding with free TM antibody molecules, the specific peak for thrombin/TM-ECD disappeared.

The force distribution histogram obtained from 1000 to 1500 force curves is shown in Figure 1B. A single maximum in the histogram by Gaussian fitting indicated that the single-molecule forces were measured. The mean value of the most probable single molecular interaction force of thrombin/TM-ECD was determined as $(45.3 \pm 1.4) \mathrm{pN}$ (from three histograms obtained in three independent experiments). According to Poisson statistics, when the possibility of rupture events is $<30 \%$, there are more than $85 \%$ probability of measuring forces between a single molecular pair [24]. As shown in Figure $1 \mathrm{C}$, the binding probability of thrombin and TM-ECD was $22.8 \% \pm 5.1 \%$. This ensured that the forces were mediated by a single pair of molecules. Blocking of the force with the TM monoclonal antibody TM-MAb solution was carried out to confirm that the measured forces were specific ones between thrombin and TM-ECD. When the binding was blocked with the TM-MAb solution, the binding probability decreased to $5.0 \% \pm 2.3 \%$, which was similar to the value when unmodified AFM tips and TM-ECD covered substrate were used.

\subsection{Effect of CSE on thrombin-TM binding on silicon substrate}

We then checked the effects of CSE on the binding of thrombin and TM-ECD. We added CSE solution into the PBS buffer to get a final concentration of 5\% CSE and incubated the silicon substrate in the above solution for $1 \mathrm{~h}$. We noticed that the binding probability of thrombin and TM-ECD decreased to $7.3 \% \pm 1.1 \%$. When further blocking with TM-MAb, the binding probability was $4.7 \% \pm 3.3 \%$ (as 


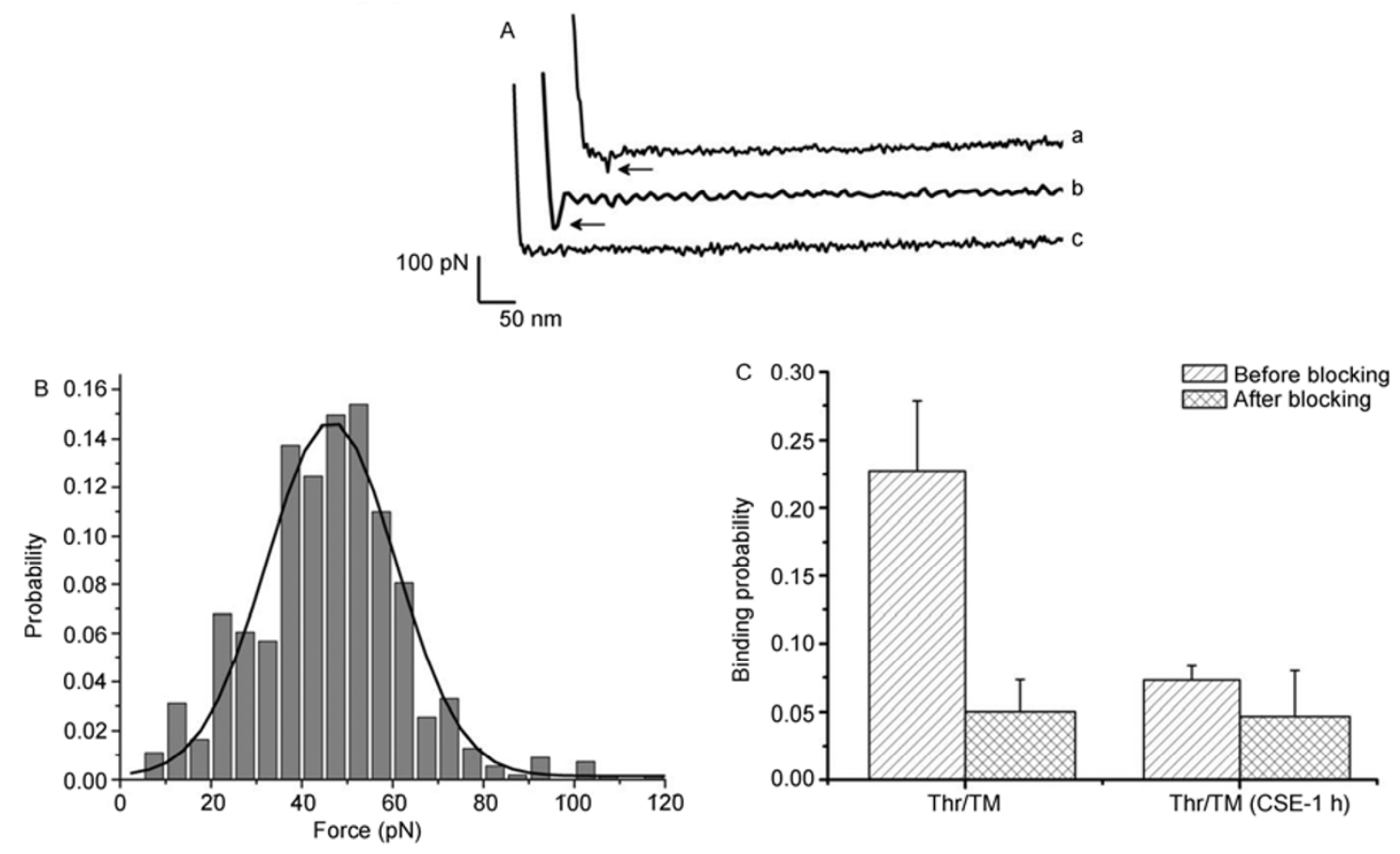

Figure 1 Binding forces measured on silicon surface. A, Representative force curves obtained with thrombin (Thr)-modified AFM tips on silicon wafers covered by the thrombomodulin extracellular domain (TM-ECD), of which (a) displays a specific binding force of TM and thrombin, (b) shows a nonspecific interaction force between the AFM cantilever and silicon substrate, and (c) is a typical force curve after the system was blocked with TM monoclonal antibody (TM-MAb) solution. The solid arrows mark the rupture of Thr/TM-ECD. B, Histogram of binding forces of Thr/TM-ECD (bars, experimental data; solid line, theoretical Gaussian distribution curve). C, Binding probabilities of thrombin (Thr)-modified AFM tips on the silicon wafer surfaces covered by TM-ECD, and the binding probability of Thr and TM-ECD repressed by 5\% CSE for $1 \mathrm{~h}$.

shown in Figure 1C). This result indicated that CSE administration indeed had effects on the interaction of thrombin and TM.

To further determine the negative effects of CSE on the binding of thrombin and TM, we measured the forces with a gradient concentration of CSE for the same incubation time. To obviously show the different effects among different concentrations of CSE, we increased the modification density of TM-ECD on silicon surfaces to obtain a relatively high binding probability of Thr/TM-ECD $(35.8 \% \pm 2.5 \%$, Figure 2A). After incubation with $0.05 \% \mathrm{CSE}$ for $1 \mathrm{~h}$, the binding probability decreased to $23.8 \% \pm 1.7 \%$. When the concentration of CSE increased to $0.5 \%$, the binding probability decreased to $15.9 \% \pm 0.8 \%$, and a higher CSE concentration of $5 \%$ led to a further decreased binding probability to $10.0 \% \pm 2.0 \%$ (Figure $2 \mathrm{~A}$ ). This suggested that CSE administration caused a lower binding probability for thrombin and TM-ECD binding, and the inhibition effect was CSE concentration dependent.

When the concentration of CSE was $0.05 \%$, the binding probability of thrombin and TM-ECD $(23.8 \% \pm 1.7 \%)$ was high enough to obtain statistical analysis of binding force, but still $<30 \%$ to ensure single-molecule interaction events. From the force curves measured with $0.05 \%$ CSE, as shown in Figure $2 \mathrm{~B}$, the force distribution histogram displayed a single maximum of $(43.8 \pm 0.4) \mathrm{pN}$ by a Gaussian fit, which was similar to the value without CSE treatment ((45.3 \pm 1.4$)$ $\mathrm{pN}$, Figure 1B). It is interesting to discover that CSE did not affect the thrombin/TM binding force, instead it reduced the binding probability.

\subsection{Measurement of single-molecule force of TM- thrombin on living cells}

TM is a membrane protein, thus we further investigated interaction of TM and thrombin with living cells, where the environment is closer to physiological conditions. Thrombin was modified on the AFM cantilever and TM was expressed on the surface of living COS-7 cells as COS-7 cells have a low level of endogenous TM. EGFP was tagged to TM, therefore the COS-7 expressing TM-EGFP or EGFP could be observed using fluorescence microscopy, and the fluorescence signal guided AFM tips to locate onto these cells for force measurements.

Force measurements were performed using the same conditions as previous experiments on silicon surfaces. The protein densities of thrombin modified on tips and TM expressing on cells were also controlled to be low enough to allow binding of single pairs of molecules. Typical force curves are shown in Figure 3A(a) and (b) for thrombin/TM interaction. When occasionally more than one pair of specific binding occurred (Figure 3A(a)), only the last peak 

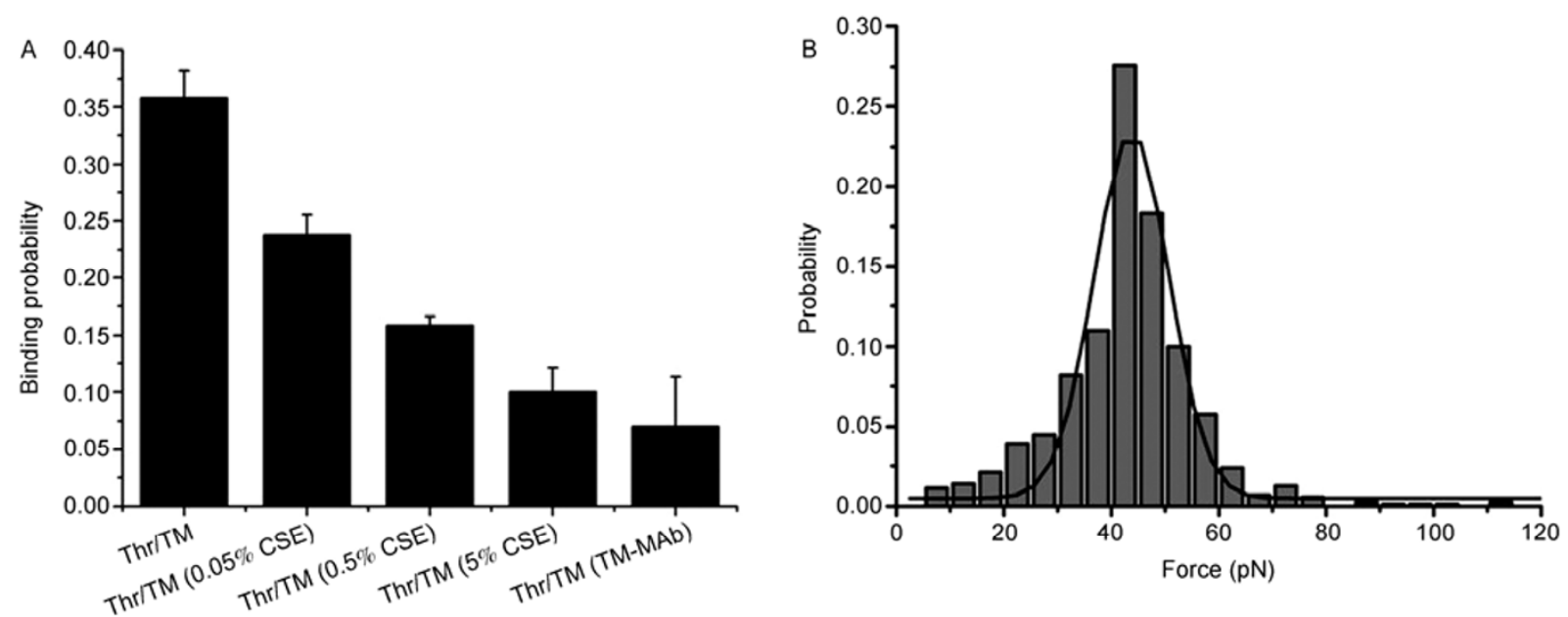

Figure 2 Binding of Thr/TM-ECD repressed by CSE on silicon surface. A, Binding probabilities of thrombin (Thr)-modified AFM tips on the silicon wafers covered by TM-ECD with different conditions (incubation with different concentrations of CSE or TM-MAb ). B, Histogram of binding forces of Thr/TM-ECD incubation with $0.05 \%$ CSE for $1 \mathrm{~h}$ (bars, experimental data; solid line, theoretical Gaussian distribution curve).
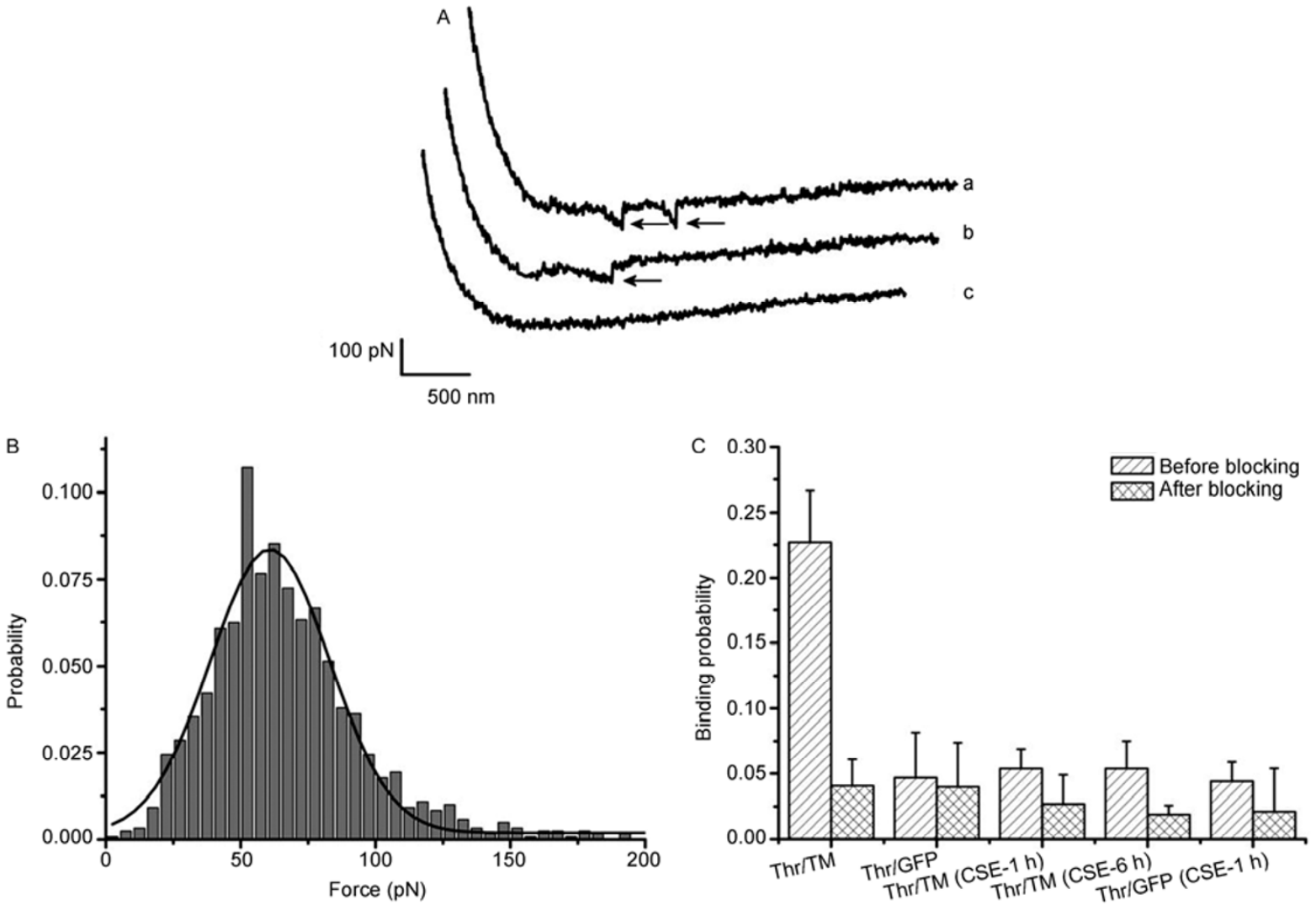

Figure 3 Binding forces measured on the living-cell surface. A, Representative force curves obtained with thrombin (Thr)-modified AFM tips on cells expressing TM-GFP (a and b), and after the system was blocked with TM-MAb solution (c). The solid arrows mark the rupture of Thr/TM. B, Histogram of binding forces of Thr/TM (bars, experimental data; solid line, theoretical Gaussian distribution curve). C, Binding probabilities of thrombin (Thr) modified tips on cells expressing different proteins under different conditions (with or without CSE).

was collected for statistical calculation. Figure $3 \mathrm{~A}(\mathrm{~b})$ is a representative force curve of single molecular rupture force of thrombin/TM. Once the TM-MAb was added in the solution, no force peak could be detected (Figure 3A(c)). Simi- lar to other reported force-distance curves on a soft substrate, such as cells $[22,28]$, the curves displayed a gradual decrease in the initial stage when the tip was retracted from the cell. The specific rupture forces appeared as the tip 
moved for a distance of $0.1-3 \mu \mathrm{m}$ due to cell elasticity, which was very different from the curves obtained on rigid substrate, as shown in Figure 1.

With 1500-2000 effective force curves obtained from several cells, we conducted statistical analysis into the binding force of thrombin/TM. The most probable single molecular interaction force of thrombin/TM is shown in Figure 3B. By averaging the mean values from the histograms of three independent experiments, the single molecule interaction force of thrombin/TM was determined as $(60.9 \pm 0.8) \mathrm{pN}$. Several control experiments were performed. When blocking thrombin/TM with the TM-MAb, the specific rupture peak disappeared for the cells expressing TM, and the binding probability decreased markedly (from $22.7 \% \pm 3.9 \%$ to $4.1 \% \pm 2.0 \%$, as shown in Figure $3 \mathrm{C}$ ). No interaction peak was observed with thrombin modified tip and the pEGFP-N1-transfected cells (without TM proteins), either before or after blocking with TM-MAb. A low binding probability of $4.7 \% \pm 3.5 \%$ was observed. The low binding probabilities shown in the above control experiments were caused by non-specific interactions between the tip and cells. These results confirmed that forces detected with the thrombin modified AFM tip on cells expressing TM were due to specific interaction between thrombin and TM. It is worth noting that the force measured with living cells was larger than that on the silicon substrate. This finding may due to the different conformations of TM-ECD in vitro and on living cells.

\subsection{Effect of CSE on TM-thrombin binding on cells}

After measuring the interaction of thrombin and TM on living cells, we studied the effect of CSE on their binding. With 5\% CSE administrated for $1 \mathrm{~h}$, the binding probability of thrombin and $\mathrm{TM}$ obviously decreased to $5.4 \% \pm 1.5 \%$ (Figure 3C). When the incubation time with 5\% CSE exposure prolonged to $6 \mathrm{~h}$, the binding probability of thrombin and TM on living cell surface was unchanged compared with the probability at $1 \mathrm{~h}(5.4 \% \pm 2.1 \%$ and $5.4 \% \pm 1.5 \%$, respectively, as shown in Figure 3C). This result indicated that CSE could disturb the binding of thrombin and TM, and the incubation time of 1 or $6 \mathrm{~h}$ did not further affect the binding of thrombin and TM induced by CSE.

In the control experiment, we added the same concentration of CSE (5\%) into cells expressing pEGFP-N1 and incubated for $1 \mathrm{~h}$. We then measured the binding of thrombin on AFM tips and cells by calculating 4000-5000 force curves from 6-7 cells. We found that either before or after blocking with TM-MAb, no obvious binding event was detected between thrombin and the cells expressing EGFP only. The results from live cell experiments demonstrated that CSE indeed inhibits the binding of thrombin and TM.

In summary, we have used AFM single molecular force spectroscopy combined with fluorescence microscopy to investigate the inhibition effects of CSE on thrombin/TM interaction both in vitro and with living cells. Our results demonstrated that CSE reduced the binding probability of thrombin to its specific receptor TM. This is the first time the interaction force of thrombin and thrombomodulin has been quantitatively assayed using AFM. The results provide new evidence for the understanding of thrombosis induced by smoking. As the CSE is a mixture of multiple components, which components can be attributed to the inhibitory effect on thrombin/TM requires further investigation.

This work was supported by the National Basic Research Program of China (Grant No. 2011CB911001), National Natural Science Foundation of China (Grant Nos. 21127901 and 21121063) and Chinese Academy of Sciences.

1 Cerami C, Founds H, Nicholl I, et al. Tobacco smoke is a source of toxic reactive glycation products. Proc Natl Acad Sci USA, 1997, 94: 13915-13920

2 Raupach T, Schäfer K, Konstantinides S, et al. Secondhand smoke as an acute threat for the cardiovascular system: a change in paradigm. Eur Heart J, 2006, 27: 386-392

3 Lavi S, Prasad A, Yang E H, et al. Smoking is associated with epicardial coronary endothelial dysfunction and elevated white blood cell count in patients with chest pain and early coronary artery disease. Circ J, 2007, 115: 2621-2627

4 Newby D E, Wright R A, Labinjoh C, et al. Endothelial dysfunction, impaired endogenous fibrinolysis, and cigarette smoking: a mechanism for arterial thrombosis and myocardial infarction. Circ J, 1999, 99: 1411-1415

5 Goto K, Nikolsky E, Lansky A J, et al. Impact of smoking on outcomes of patients with ST-segment elevation myocardial infarction (from the HORIZONS-AMI trial). Am J Cardiol, 2011, 108: 1387-1394

6 Salomaa V, Matei C, Aleksic N, et al. Soluble thrombomodulin as a predictor of incident coronary heart disease and symptomless carotid artery atheroscierosis in the Atherosclerosis Risk in Communities (ARIC) Study: a case-cohort study. Lancet, 1999, 353: 1729-1734

7 Barua R S, Ambrose J A, Saha D C, et al. Smoking is associated with altered endothelial-derived fibrinolytic and antithrombotic factors an in vitro demonstration. Circ J, 2002, 106: 905-908

8 Pittilo M. Cigarette smoking, endothelial injury and cardiovascular disease. Int J Exp Pathol, 2000, 81: 219-230

9 Bernhard D, Csordas A, Henderson B, et al. Cigarette smoke metal-catalyzed protein oxidation leads to vascular endothelial cell contraction by depolymerization of microtubules. FASEB J, 2005, 19: 1096-1107

10 Nakamura Y, Romberger D J, Tate L, et al. Cigarette smoke inhibits lung fibroblast proliferation and chemotaxis. Am J Resp Crit Care, 1995, 151: 1497-1503

11 Su Y, Han W, Giraldo C, et al. Effect of cigarette smoke extract on nitric oxide synthase in pulmonary artery endothelial cells. Am J Resp Cell Mol, 1998, 19: 819-825

12 Edirisinghe I, Arunachalam G, Wong C, et al. cigarette smoke-induced oxidative/nitrosative stress impairs vegf-and fluid shear stress-mediated signaling in endothelial cells. Antioxid Redox Signal, 2010, 12: 1355-1369

13 Hu X, Ma Y, Wang C, et al. Effects of simvastatin on cigarette smoke extract induced tissue-type plasminogen activator and plasminogen activator inhibitor-1 expression in human umbilical vein endothelial cells. Chinese Med J-Peking, 2009, 122: 2380-2385

14 Cacciola R R, Guarino F, Polosa R. Relevance of endothelial-haemostatic dysfunction in cigarette smoking. Curr Med Chem, 2007, 14: 1887-1892

15 Dahlbäck B, Villoutreix B O. Regulation of blood coagulation by the 
protein C anticoagulant pathway. Arteriosclerosis, 2005, 25: 1311-1320

16 Bajzar L. Thrombin activatable fibrinolysis inhibitor and an antifibrinolytic pathway. Arteriosclerosis, 2000, 20: 2511-2518

17 Van de Wouwer M, Collen D, Conway E M. Thrombomodulinprotein C-EPCR system. Arteriosclerosis, 2004, 24: 1374-1383

18 Clausen-Schaumann H, Seitz M, Krautbauer R, et al. Force spectroscopy with single bio-molecules. Curr Opin Chem Biol, 2000, 4: 524-530

19 Xu Y, Shi X, Fang X. Biomolecular interaction study by atomic force microscopy single-molecule force spectroscopy. Chin Bull Life Sci, 2008, 20: 39-45

20 Shi X, Xu L, Yu J, et al. Study of inhibition effect of Herceptin on interaction between Heregulin and ErbB receptors HER3/HER2 by single-molecule force spectroscopy. Exp Cell Res, 2009, 315: 2847-2855

21 Yang H, Yu J, Fu G, et al. Interaction between single molecules of Mac-1 and ICAM-1 in living cells: an atomic force microscopy study. Exp Cell Res, 2007, 313: 3497-3504

22 Yang Y, Xu Y, Xia T, et al. A single-molecule study of the inhibition effect of Naringenin on transforming growth factor- $\beta$ ligand-receptor binding. Chem Commun, 2011, 47: 5440-5442

23 Yu J, Sun S, Jiang Y, et al. Single molecule study of binding force between transcription factor TINY and its DNA responsive element. Polym J, 2006, 47: 2533-2538

24 Yu J, Wang Q, Shi X, et al. Single-molecule force spectroscopy study of interaction between transforming growth factor $\beta 1$ and its receptor in living cells. J Phys Chem B, 2007, 111: 13619-13625

25 Li Y, Shi X L, Liu H L, et al. Study of the effect of atorvastatin on the interaction between ICAM-1 and CD11b by live-cell single-molecule force spectroscopy. Sci China Chem, 2010, 53: $752-758$

26 Hoshino Y, Mio T, Nagai S, et al. Cytotoxic effects of cigarette smoke extract on an alveolar type II cell-derived cell line. Am J Physiol-Lung C, 2001, 281: L509-L516

27 Laurent P, Janoff A, Kagan H. Cigarette smoke blocks cross-linking of elastin in vitro. Am Rev Resp Dis, 1983, 127: 189-192

28 Benoit M, Gabrie D, Gerisch G, et al. Discrete interactions in cell adhesion measured by single-molecule force spectroscopy. Nat Cell Biol, 2000, 2: 313-317

Open Access This article is distributed under the terms of the Creative Commons Attribution License which permits any use, distribution, and reproduction in any medium, provided the original author(s) and source are credited. 